\title{
Heartbeat: cardiovascular disease risk and reproductive factors
}

The likelihood of cardiovascular disease (CVD) in both women and men largely is explained by well-known lifestyle and clinical risk factors. Several studies have suggested that a woman's reproductive history also might affect the risk of CVD, but results have been inconsistent and methodology suboptimal. In this issue of Heart, Peters and Woodward ${ }^{1}$ report the association between several reproductive factors and subsequent incident CVD over 7 years of follow-up in 482,000 participants in the UK Biobank study with CVD defined as incident myocardial infarction (fatal or non-fatal) or stroke. ${ }^{1}$ The risk of CVD was increased in women with early menarche ( $<12$ years), early menopause $(<47$ years), younger age at first birth, or a history of miscarriage, stillbirth or hysterectomy (figure 1). Interestingly, in both men and women, each additional child was associated with an increased risk of CVD with an HR of 1.03 (1.00 to 1.06$)$ in women and 1.03 (1.02 to 1.05 ) in men.

Unfortunately, the question of whether any of these associations are causal remains unanswered. For example, the conventional hypothesis that hormonal changes at menopause are the cause of increased CVD risk might not be correct. As the authors note: "It has also been suggested that it is not menopause that adversely affects cardiovascular risk but rather that cardiovascular risk factors determine the age at menopause, possibly through direct effects on the endocrine system or by inducing ischaemic damage in the ovaries." They go on to recommend that further studies are needed on each of these reproductive factors to identify potential biological, behavioural or social mechanisms that might explain the association with CVD risk.

Prevention of stroke in patients with atrial fibrillation (AF) is of paramount importance, particularly in patients with heart failure. Despite convincing evidence and guideline recommendations supporting use of oral

Correspondence to Professor Catherine M Otto, Division of Cardiology, University of Washington, Seattle,WA 98195, USA; cmotto@uw.edu
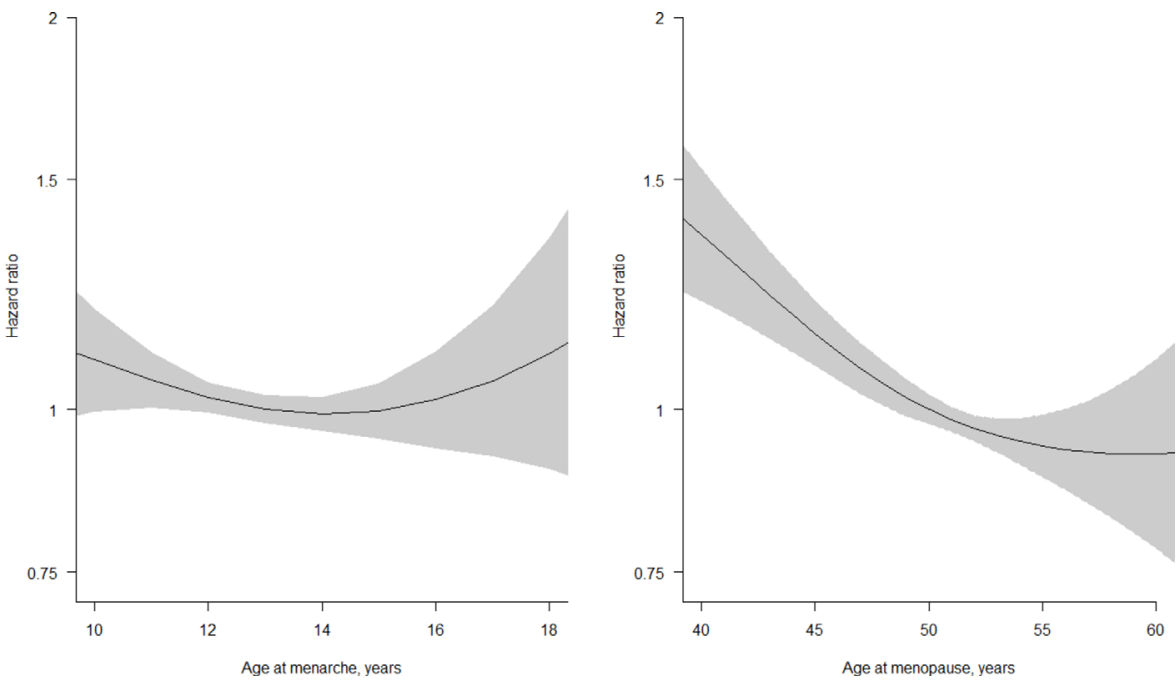

Figure 1 Penalised spline plots with adjusted HRs $(95 \% \mathrm{Cl})$ for cardiovascular disease associated with women's age at menarche and age at natural menopause. Analyses are adjusted for age, Townsend deprivation index, smoking status, systolic blood pressure, history of diabetes and body mass index.

anticoagulation (OAC) for this indication, many patients are not on effective therapy. In a study by Savarese and colleagues ${ }^{2}$ that included over 21000 patients with both heart failure and AF in Sweden, only 58\% received OAC. Encouragingly the likelihood of OAC treatment was higher with a higher $\mathrm{CHA}_{2} \mathrm{DS}_{2}$-VASc score and OAC use was associated with fewer adverse events. However, underuse of OAC was associated with several factors that actually increase risk of stroke including older age, paroxysmal AF, inappropriate use of anti-platelet therapy and more severe heart failure. Other associations with underuse of OAC, such as follow-up by primary care, living alone and lower income suggest inequities in provision of healthcare services.

In addition, as Cowie and Zakeri' ${ }^{3}$ comment in an editorial: 'The chance of being anticoagulated did rise as the CHA2DS2-VASc score rose, but at a less steep gradient than the chance dropped as the HAS-BLED score rose. In other words, physicians were more influenced by a rise in bleeding risk than they were by a rise in stroke risk. This example of omission bias is well known and is perhaps explained by physicians still being of the mindset that if a stroke occurs, it can be considered 'natural' whereas causing a bleed by anticoagulation is iatrogenic and therefore more likely to be perceived as their 'fault'.' In order to overcome this bias, Cowie and Zakeri recommend: 'As international guidelines suggest, we should ensure we detect atrial fibrillation (whether persistent or paroxysmal), make an objective assessment of both the stroke and bleeding risk using an accepted tool, and then seek to have a discussion tailored to the individual patient so that a shared decision can be made on the best way forward for that individual.'

Resting heart rate (RHR) is a simple measure that reflects physical fitness and cardiovascular health. Based on data from over 40000 patients in the Melbourne Collaborative Cohort Study, Seviiri and colleagues ${ }^{4}$ found that both RHR, and the change in RHR over a decade, were associated with an increased risk of death from CVD, cancer or other causes (figure 2).

In the accompanying editorial, Nanchen ${ }^{5}$ suggests "Even if causality is not yet established, clinicians should now screen for abnormal RHR defined as above $70 \mathrm{bpm}$ for men and above $80 \mathrm{bpm}$ 

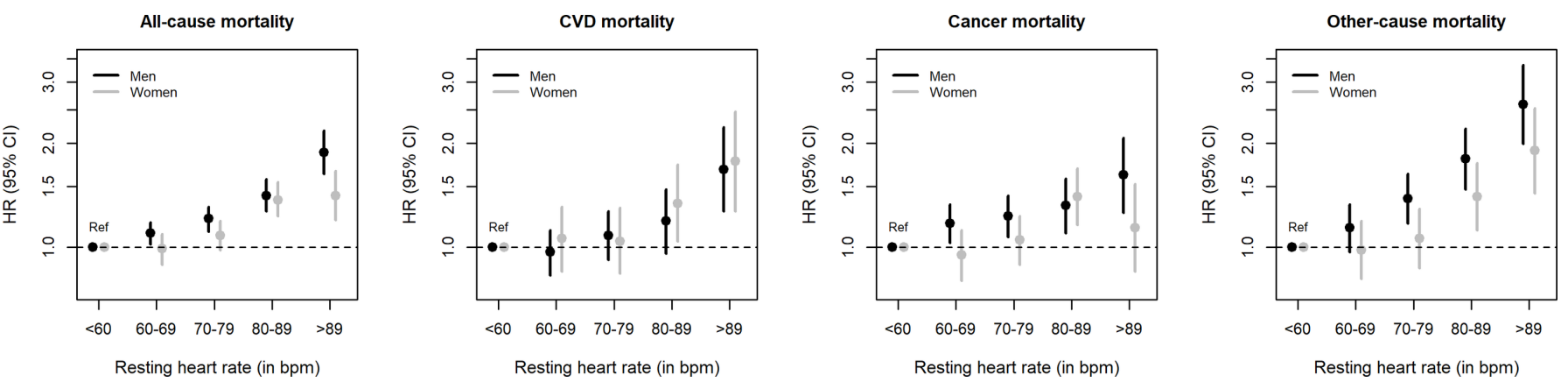

Figure 2 Association of resting heart rate measured at baseline with all-cause and cause-specific mortality, stratified by sex. Cox models were adjusted for age, country of birth, level of education, waist circumference, alcohol consumption, smoking, physical activity score, alternate healthy eating index, total serum cholesterol, sodium-potassium ratio, caffeine, blood pressure, history of hypertension, angina, asthma and diabetes. Interactions between sex and RHR were tested by comparing models with and without interaction terms between sex and RHR, using likelihood ratio tests. $P$ values for interaction: all-cause mortality: $p=0.07$; in the $90+$ bpm category: $p=0.02$; cancer mortality: $p=0.72 ; C V D$ mortality: $p=0.77 ;$ othercause mortality: $p=0.55$. bpm, beats per minute; $C V D$, cardiovascular disease; RHR, resting heart rate.

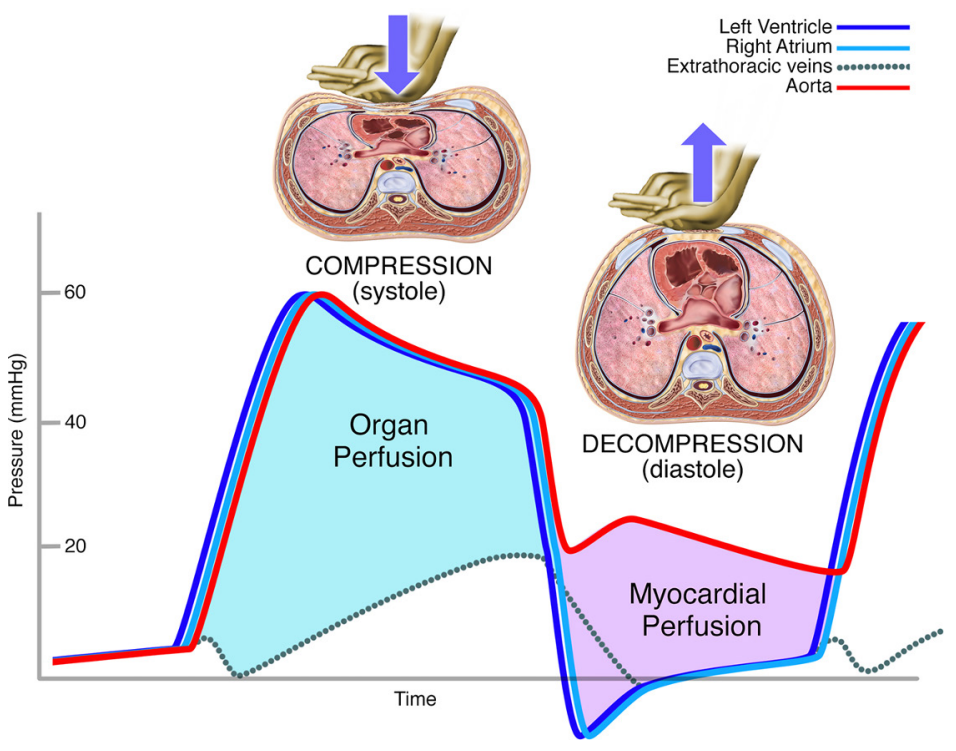

Figure 3 Haemodynamic effects of compression and decompression phases of cardiopulmonary resuscitation. Compression phase creates organ perfusion pressure (difference between aortic and extrathoracic vein pressure). Decompression phase creates myocardial perfusion pressure (difference between aortic and right atrial pressure). Data adapted from Criley et al. ${ }^{8}$

\section{Exercise Paradox}

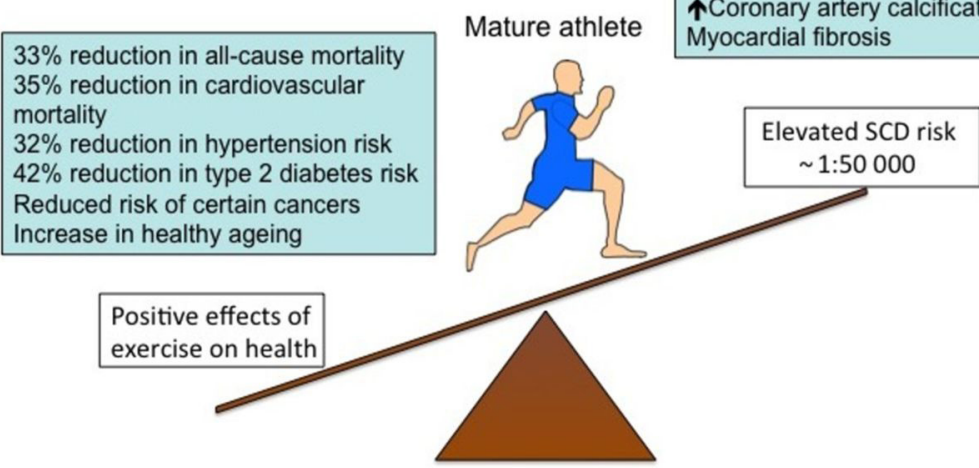

Figure 4 Exercise paradox. AF, atrial fibrillation; SCD, sudden cardiac death. for women when they monitor blood pressure in healthy adults. Because it is unknown if targeting RHR using drugs such as beta-blockers or ivabradine will improve prognosis, the most appropriate preventive attitude towards high RHR values may be lifestyle counselling."

All healthcare providers now consider training in cardiopulmonary resuscitation (CPR) a core competency for patient care. Yet, the effectiveness of CPR varies widely around the world. In a concise review article, entitled 'Cardiopulmonary resuscitation: the science behind the hands', Harris and Kudenchuk ${ }^{6}$ summarise the evidence underlying current recommendations for performing CPR and provide clear explanations of why CPR works (figure 3). Hopefully understanding how it works might improve CPR performance and, ultimately, clinical outcomes.

The Education in Heart article in this issue provide an educational review for management of mature athletes, ${ }^{7}$ including a discussion of the benefits and risks associated with regular high-intensity exercise (figure 4).

\section{Competing interests None declared.}

Patient consent Not required.

Provenance and peer review Commissioned; internally peer reviewed.

(c) Article author(s) (or their employer(s) unless otherwise stated in the text of the article) 2018. All rights reserved. No commercial use is permitted unless otherwise expressly granted.

\section{(D) Check for updates}

To cite Otto CM. Heart 2018;104:1045-1047.

Heart 2018;104:1045-1047

doi:10.1136/heartjnl-2018-313503 


\section{REFERENCES}

1 Peters SA, Woodward M. Women's reproductive factors and incident cardiovascular disease in the UK Biobank. Heart 2018;104:1069-75.

2 Savarese G, Sartipy U, Friberg L, et al. Reasons for and consequences of oral anticoagulant underuse in atrial fibrillation with heart failure. Heart 2018;104:1093-100.
3 Cowie MR, Zakeri R. Preventing stroke in patients with heart failure: why are patients losing out? Heart 2018;104:1050-2.

4 Seviiri M, Lynch BM, Hodge AM, et al. Resting heart rate, temporal changes in resting heart rate, and overall and cause-specific mortality. Heart 2018; 104:1076-85.

5 Nanchen D. Resting heart rate: what is normal? Heart 2018;104:1048-9.
6 Harris AW, Kudenchuk PJ. Cardiopulmonary resuscitation: the science behind the hands. Heart 2018;104:1056-61.

7 D'Silva A, Sharma S. Management of mature athletes with cardiovascular conditions. Heart 2018;104:1125-34.

8 Criley JM, Niemann JT, Rosborough JP, et al. Modifications of cardiopulmonary resuscitation based on the cough. Circulation 1986;74(suppl 4):42-50. 